\title{
A THEOREM IN THE GEOMETRY OF MATRICES
}

\section{JOSEPHINE MITCHELL}

The following theorem is of interest in that it shows a connection between a result due to Hua in the geometry of matrices with one due to Bergman in several complex variables. Let $Z=\left(z_{j k}\right)(j=1, \cdots, m$; $k=1, \cdots, n)$ be a matrix of signature $(m, n)$ (that is, of $m$ rows and $n$ columns), $\bar{Z}^{\prime}=\left(\bar{z}_{k j}\right)$ its conjugate transposed matrix, $I$ the identity matrix, and 0 the zero matrix. We consider the domain defined by $I-Z \bar{Z}^{\prime}>0$, consisting of all points $Z$ with $m n$ complex coordinates $\left(z_{11}, z_{12}, \cdots, z_{m n}\right)$ for which the Hermitian quadratic form,

$$
\sum_{j, k=1}^{m}\left(\delta_{j k}-\sum_{i=1}^{n} z_{j i} \bar{z}_{k i}\right) x_{j} \bar{x}_{k},
$$

is positive definite in the auxiliary variables $\left(x_{1}, \cdots, x_{n}\right)$. The domain $I-Z \bar{Z}^{\prime}>0$ lies in $2 m n$-dimensional Euclidean space and is a certain generalization of the unit circle in the complex $z$-plane.

We prove that:

THEOREM. The positive definite quadratic differential form

$$
Q \equiv \sum_{j, p=1}^{m} \sum_{k, q=1}^{n} \frac{\partial^{2} \log \left[\operatorname{det}\left(I-Z \bar{Z}^{\prime}\right)\right]^{-1}}{\partial z_{j k} \partial \bar{z}_{p q}} d z_{j k} d \bar{z}_{p q}
$$

defines a metric in the domain $I-Z \bar{Z}^{\prime}>0$ which is invariant with respect to the conjunctive group of signature $(m, n)$.

The conjunctive group of signature $(m, n)$ is the set of matrices $T$ such that $\bar{T} F T^{\prime}=F$, where

$$
F=\left(\begin{array}{rr}
I & 0 \\
0 & -I
\end{array}\right) \text { and } T=\left(\begin{array}{ll}
A & B \\
C & D
\end{array}\right) .
$$

Proof of The Theorem. Hua has proved that the positive definite quadratic differential form $\sigma\left(\left(I-Z \bar{Z}^{\prime}\right)^{-1} d Z\left(I-\bar{Z}^{\prime} Z\right)^{-1} d \bar{Z}^{\prime}\right)$, where $\sigma(Z)$ is the trace of matrix $Z$ and $d Z$ its differential, is invariant with respect to the conjunctive group of signature $(m, n)$ [On the theory of automorphic functions of a matrix variable I, Amer. J. Math. vol. 66 (1944) pp. 470-488]. We prove the theorem by proving the following formula, using techniques due to Hua

$$
\text { (A) } Q=\sigma\left(\left(I-Z \bar{Z}^{\prime}\right)^{-1} d Z\left(I-\bar{Z}^{\prime} Z\right)^{-1} d \bar{Z}^{\prime}\right) \text {. }
$$

Presented to the Society, February 25, 1950; received by the editors February 23, 1950. 
Now $d(\operatorname{det} X)=\sum_{k=1}^{n} d x_{1 k} X_{1 k}+\cdots+\sum_{k=1}^{n} d x_{n k} X_{n k}=\sigma\left(d X X^{*}\right)$, where $X_{j k}$ is the cofactor of $x_{j k}$ in the matrix $X$ and $X^{*}$ is the adjoint matrix. Also, for nonsingular matrices $X X^{*}=I \operatorname{det} X$ or $X^{*}$ $=(\operatorname{det} X) X^{-1}$. Hence, since $\sigma(k A)=k \sigma(A)$ for $k$ scalar,

$$
d(\operatorname{det} X)=\operatorname{det} X \sigma\left(d X X^{-1}\right),
$$

and

$$
d(\log \operatorname{det} X)=\sigma\left(d X X^{-1}\right) .
$$

We now form $Q$ in two steps. Using (2), we have

$$
\begin{aligned}
-\sum_{p=1}^{m} \sum_{q=1}^{n} \frac{\partial}{\partial \bar{z}_{p q}} \log \operatorname{det}\left(I-Z \bar{Z}^{\prime}\right) d \bar{z}_{p q} & =-d \bar{Z}\left(\log \operatorname{det}\left(I-Z \bar{Z}^{\prime}\right)\right) \\
& =-\sigma\left(d \bar{Z}\left(I-Z \bar{Z}^{\prime}\right)\left(I-Z \bar{Z}^{\prime}\right)^{-1}\right) \\
& =\sigma\left(Z d \bar{Z}^{\prime}\left(I-Z \bar{Z}^{\prime}\right)^{-1}\right) ;
\end{aligned}
$$

whence

$$
Q=-\sum_{j=1}^{m} \sum_{k=1}^{n} \frac{\partial}{\partial z_{j k}}\left[\sum_{p=1}^{m} \sum_{q=1}^{n} \frac{\partial}{\partial \bar{z}_{p q}} \log \operatorname{det}\left(I-Z \bar{Z}^{\prime}\right) d \bar{z}_{p q}\right] d z_{j k}
$$

(4)

$$
=\sum_{j=1}^{m} \sum_{k=1}^{n} \frac{\partial}{\partial z_{j k}} \sigma\left(Z d \bar{Z}^{\prime}\left(I-Z \bar{Z}^{\prime}\right)^{-1}\right) d z_{j k} .
$$

Writing $X=Z d \bar{Z}^{\prime}, \quad Y=\left(I-Z \bar{Z}^{\prime}\right)^{-1}$ and using the definition of $\sigma(A)$, we have

$$
\begin{aligned}
Q & =\sum_{i, k} \frac{\partial}{\partial z_{j k}} \sigma(X Y) d z_{j k} \\
& =\sum_{j, k} \frac{\partial}{\partial z_{j k}}\left(\sum_{\alpha, \beta} x_{\alpha \beta} y_{\beta \alpha}\right) d z_{j k}\left(X=\left(x_{\alpha \beta}\right), Y=\left(y_{\alpha \beta}\right)\right)
\end{aligned}
$$

(5)

$$
\begin{aligned}
& =\sum_{j, k} \sum_{\alpha, \beta}\left(\frac{\partial x_{\alpha \beta}}{\partial z_{j k}} y_{\beta \alpha}+x_{\alpha \beta} \frac{\partial y_{\beta \alpha}}{\partial z_{j k}}\right) d z_{j k} \\
& =\sum_{\alpha, \beta}\left[\left(\sum_{i, k} \frac{\partial x_{\alpha \beta}}{\partial z_{j k}} d z_{j k}\right) y_{\beta \alpha}+x_{\alpha \beta}\left(\sum_{i, k} \frac{\partial y_{\beta \alpha}}{\partial z_{j k}} d z_{j k}\right)\right] \\
& =\sum_{\alpha, \beta}\left(d x_{\alpha \beta} y_{\beta \alpha}+x_{\alpha \beta} d y_{\beta \alpha}\right) \\
& =\sigma(d X Y+X d Y) .
\end{aligned}
$$


But $d X=d Z d \bar{Z}^{\prime}$ and $d Y=-\left(I-Z \bar{Z}^{\prime}\right)^{-1}(-d Z) \bar{Z}^{\prime}\left(I-Z \bar{Z}^{\prime}\right)^{-1}$ so that

$$
\begin{aligned}
Q & =\sigma\left(d Z d \bar{Z}^{\prime}\left(I-Z \bar{Z}^{\prime}\right)^{-1}+Z d \bar{Z}^{\prime}\left(I-Z \bar{Z}^{\prime}\right)^{-1} d Z \bar{Z}^{\prime}\left(I-Z \bar{Z}^{\prime}\right)^{-1}\right) \\
& =\sigma\left(d Z d \bar{Z}^{\prime}\left(I-Z \bar{Z}^{\prime}\right)^{-1}+d Z \bar{Z}^{\prime}\left(I-Z \bar{Z}^{\prime}\right)^{-1} Z d \bar{Z}^{\prime}\left(I-Z \bar{Z}^{\prime}\right)^{-1}\right),
\end{aligned}
$$

since $\sigma(A+B)=\sigma(A)+\sigma(B)$ and $\sigma(A B)=\sigma(B A)$, and this latter term equals

$$
\left(d Z\left[I+\bar{Z}^{\prime}\left(I-Z \bar{Z}^{\prime}\right)^{-1} Z\right] d Z^{\prime}\left(I-Z \bar{Z}^{\prime}\right)^{-1}\right) .
$$

From the identity $I+\bar{Z}^{\prime}\left(I-Z \bar{Z}^{\prime}\right)^{-1} Z=\left(I-\bar{Z}^{\prime} Z\right)^{-1}$ this gives

$$
\begin{aligned}
Q & =\sigma\left(d Z\left(I-\bar{Z}^{\prime} Z\right)^{-1} d \bar{Z}^{\prime}\left(I-Z \bar{Z}^{\prime}\right)^{-1}\right) \\
& =\sigma\left(\left(I-Z \bar{Z}^{\prime}\right)^{-1} d Z\left(I-\bar{Z}^{\prime} Z\right)^{-1} d \bar{Z}^{\prime}\right),
\end{aligned}
$$

which proves formula (A).

Bergman proved the following theorem. Let $K\left(z_{1}, \cdots, z_{\kappa}\right.$; $\bar{z}_{1}, \cdots, \bar{z}_{\kappa}$ ) be the kernel function of a finite schlicht domain lying in $2 \kappa$ dimensional Euclidean space. Then the Hermitian differential form

$$
\sum_{\mu, \nu=1}^{\kappa} \frac{\partial^{2} \log K\left(z_{1}, \cdots, z_{\kappa} ; \bar{z}_{1}, \cdots, \bar{z}_{\kappa}\right) d z_{\mu} d \bar{z}_{\nu}}{\partial z_{\mu} \partial \bar{z}_{\nu}}
$$

defines a positive definite metric which is invariant with respect to pseudo-conformal transformations. [Sur les fonctions orthogonales de plusieurs variables complexes, Mémorial des Sciences Mathématiques, vol. 106, 1947].

A comparison of the two theorems leads us to suspect that the function $V^{-1}\left[\operatorname{det}\left(I-Z \bar{Z}^{\prime}\right)\right]^{-m-n}$, where $V$ is the Euclidean volume of the domain $I-Z \bar{Z}^{\prime}>0$, is the Bergman kernel function of the domain $I-Z \bar{Z}^{\prime}>0$. We have verified this supposition in the case of matrices of signature $(2,2)$ by the classical method of constructing a complete orthonormal system $\left\{\phi_{p}(Z)\right\}(p=1,2, \cdots)$ of functions, analytic and Lebesgue square integrable on $I-Z \bar{Z}^{\prime}>0$, and showing that the absolutely and uniformly convergent series $\sum_{p=1}^{\infty} \phi_{p}(Z) \Phi_{p}(Z)$ has the sum $\left(12 \pi^{4}\right)^{-1}\left[\operatorname{det}\left(I-Z \bar{Z}^{\prime}\right)\right]^{-4}$ [To appear in the Proceedings of the Third Canadian Mathematical Congress, Vancouver, 1949].

UNIVERSITY OF ILLINOIS 